\title{
Rightward hemispheric asymmetries in auditory language cortex in children with autistic disorder: an MRI investigation
}

\author{
Nicole M. Gage • Jenifer Juranek • Pauline A. Filipek • \\ Kathryn Osann • Pamela Flodman • \\ A. Lisette Isenberg • M. Anne Spence
}

Received: 5 January 2009 /Accepted: 6 March 2009/Published online: 4 April 2009

(C) The Author(s) 2009. This article is published with open access at Springerlink.com

\begin{abstract}
Purpose: determine if language disorder in children with autistic disorder (AD) corresponds to abnormalities in hemispheric asymmetries in auditory language cortex. Methods: MRI morphometric study in children with AD $(n=50)$ to assess hemispheric asymmetries in auditory language cortex. A key region of interest was the planum temporale (PT), which is larger in the left hemisphere in most healthy individuals. Results: (i) Heschl's gyrus and planum polare showed typical hemisphere asymmetry patterns; (ii) posterior Superior Temporal Gyrus (pSTG) showed significant rightward asymmetry; and (iii) PT showed a trend for rightward asymmetry that was significant when constrained to right-handed boys $(n=30)$. For right-handed boys, symmetry indices for pSTG were significantly positively correlated with those for PT. PT asymmetry was age dependent, with greater rightward
\end{abstract}

Electronic supplementary material The online version of this article (doi:10.1007/s11689-009-9010-2) contains supplementary material, which is available to authorized users.

N. M. Gage $(\square) \cdot$ A. L. Isenberg

Department of Cognitive Sciences, University of California,

Irvine 92697-5100 CA, USA

e-mail: ngage@uci.edu

J. Juranek

Department of Pediatrics, Health Science Center at Houston,

The University of Texas,

Houston, TX, USA

P. A. Filipek $\cdot$ P. Flodman $\cdot$ M. A. Spence

Department of Pediatrics, University of California,

Irvine, CA, USA

K. Osann

Department of Medicine, University of California,

Irvine, CA, USA asymmetry with age. Conclusions: results provide evidence for rightward asymmetry in auditory association areas (pSTG and PT) known to subserve language processing. Cumulatively, our data provide evidence for a differing maturational path for PT for lower functioning children with $\mathrm{AD}$, with both pre- and post-natal experience likely playing a role in PT asymmetry.

Keywords Auditory cortex · Developmental disorder . Heschl's gyrus · Language cortex · Posterior superior temporal gyrus

\section{Introduction}

Language impairment is a defining feature of autistic disorder (AD) [1], and though it is well documented in the literature through clinical observations, the behavioral description is incomplete and the neural bases remain largely unknown. An important question in autism research is to what extent language dysfunction stems from functional abnormalities, structural anomalies in cortical language systems, or both.

Functionally, language systems are left-lateralized for most right-handed individuals [2]. Less is known about the developmental of language lateralization, however an fMRI study provided evidence for a leftward bias in speech processing in infants (3 months) [3]. There is evidence for a developmental trajectory throughout childhood, with language processing becoming more left-lateralized with age [4], becoming strongly left-dominant by age 7 [5-7; for a review see 8]. Investigations of language lateralization in individuals with $\mathrm{AD}$ present a different picture: evidence for anomalous or rightward language dominance in $\mathrm{AD}$ has 
been provided using behavioral, electrophysiological, and functional neuroimaging techniques [9-17]. Flagg and colleagues [4] reported rightward trajectory for language dominance as a function of age for children with $\mathrm{AD}$, indicating that experience may play a role in language laterality. Cumulatively, these studies provide evidence that the functional development of cortical language systems follows a different maturational path in $\mathrm{AD}$. If this is the case, an open but important question is whether this rightward trajectory reflects experience-driven abnormalities in the functional development of language, structurallybased anomalies in cortical language regions, or both.

While there have been many studies investigating functional language in $\mathrm{AD}$, few studies have assessed brain structures for language processing: just 3 studies have used anatomical magnetic resonance imaging (aMRI) segmentation and parcellation techniques to quantify hemisphere asymmetries in language regions in children with $\mathrm{AD}$. A key region of interest (ROI) in these studies was planum temporale (PT), a region in perisylvian cortex that is larger in the left hemisphere in most healthy right-handed individuals [18]. In the first study using aMRI to evaluate language areas in children with $\mathrm{AD}$, Herbert and colleagues [19] measured volumetric hemispheric asymmetries in high functioning (HF) boys with AD (7-11 years) and agematched typically developing (TD) controls. They calculated a hemispheric symmetry index (SI) for each brain ROI and reported greater PT leftward asymmetry in AD. While this effect reached statistical significance, there was substantial variability, with both groups' SI values spanning zero. A second finding was a trend towards rightward asymmetry in posterior Superior Temporal Gyrus (pSTG) for both groups.

Using similar (but not identical) aMRI volumetric analyses, Rojas and colleagues investigated hemispheric asymmetries in Heschl's gyrus (HG) and PT in HF children with AD (5-16 years) and age-matched TD controls [20]. They reported no group differences for HG, with a trend towards left > right HG in both groups, and lack of PT asymmetry for $\mathrm{AD}$, reflecting smaller left rather than a larger right PT. No significant correlations for age, education, handedness, or full scale IQ were found. They reported a similar effect in a study with adults with $\mathrm{AD}$, providing evidence for typical HG hemispheric asymmetries and a sharp lack of PT asymmetry in individuals with AD [20, 21]. In a study by De Fosse and colleagues [22] hemispheric asymmetries in cortical language regions were investigated in boys (7-11 years) with $\mathrm{AD}$ and TD controls. A priori, they separated the AD sample into two groups: those with language impairment (LI) (AD-LI) and without (AD-N) and calculated SI's for PT and pSTG. AD-LI showed significantly leftward PT asymmetry, however neither the AD-N nor the TD group had significant leftward
PT asymmetries. There was a trend for rightward pSTG asymmetry for both $\mathrm{AD}$ groups.

The inconsistent findings across these three studies are not surprising due to the high variability observed in individuals on the autistic spectrum on almost any brain measure. Additionally, each study had a relatively small sample of relatively HF individuals with AD. It may be the case that asymmetries in cortical language structures differ according to level of language impairment. This is suggested by the findings of De Fosse and colleagues [22], where PT asymmetries differed in scale for children with AD-LI compared to AD-N. The mixed results found in these studies combined with the small samples motivate a study with a large sample in order to shed more light on hemispheric asymmetries in auditory language cortex.

In this investigation, we compared quantitative volumetric measurements of auditory and language regions to assess hemispheric asymmetries in 4 ROIs (planum polare (PP), HG, PT, and pSTG) in children with AD. aMRI analyses used were nearly identical to those in the studies by Herbert and De Fosse [19, 22], allowing us to directly compare our findings with their results. A key goal was to assess the correspondence between asymmetries and gender, handedness, and age. Based on the findings of De Fosse and colleagues [22], we assessed correspondence between neuropsychological language measures (verbal IQ (VIQ) and Peabody Picture Vocabulary Test (PPVT)) and PT asymmetries to determine whether degree of asymmetry related to level of impairment. Three sets of monozygotic (MZ, identical) twins were included in our sample. Volumes of the ROIs were measured for these children and results provided in a Supplementary figure (Supplementary Fig. 6) for descriptive purposes.

\section{Materials and methods}

\section{Study sample}

Sixty-five participants in the UCI Autism Research Project were assessed with a battery of standardized neuropsychological tests and structural neuroimaging as part of a largescale, multi-site collaborative study (Collaborative Program for Excellence in Autism (CPEA)). Nine children who did not meet research criteria for $\mathrm{AD}$ and three children with identified chromosome abnormalities were excluded from analysis. Our sample included three pairs of MZ twins: one of each pair was randomly excluded, leaving a final sample of 50. Children were tested using the Autism Diagnostic Observation Schedule (ADOS; [23]) and the Autism Diagnostic Interview-Revised (ADI-R; [24]). Forty-six children met all ADOS and ADI criteria for autistic disorder. Four additional children with a clinical diagnosis 
of $\mathrm{AD}$ were included. Two of these children met all ADOS and ADI criteria with the exception of the stereotypic behavior domain of the ADI; two additional children met all ADI criteria and two of the three ADOS cutoffs but were one point below cutoff for the third ADOS domain.

Handedness was determined for 43 of the 50 children through neurological exam records or parent interviews using a modified Edinburgh Handedness Inventory [25]. Thirty males and six females were righthanded, four males were left-handed and three children (two males, one female) used both hands equally. The subgroup of right-handed males resembled the larger group with respect to age, ADOS, ADI, IQ and language testing (Table 1).

Standard scores are reported for cognitive ability (Stanford Binet Intelligence Test, [26]; or Mullen Scales of Early Learning, [27]), adaptive behavior (Vineland Adaptive Behavior Scales-Revised (VABS); [28]), and for expressive and receptive language (Expressive One-Word Picture Vocabulary Test (EOWPVT), [29]; Peabody Picture Vocabulary Test (PPVT, [30])). Standard scores are based on a mean score of 100 with standard deviation of 15 for TD children.

\section{MRI acquisition}

MRI scans were performed on the Picker International 1.5 Tesla system located at UCI Medical Center. After conventional sagittal scout and coronal $\mathrm{T}_{2}$-weighted sequences, a sagittal 3D-FLASH T1-weighted spoiled gradient echo pulse sequence was performed with the following pulse sequence parameters: TR/TE $=22 / 10 \mathrm{~ms}$; Flip Angle $=30^{\circ}$; $\mathrm{FOV}=256 \times 256 \mathrm{~mm}$; Matrix $=256 \times 256$; Slice thickness $=$ $1.20 \mathrm{~mm}$ (145 contiguous sections); Inplane pixel dimensions $(x, y)=0.976562 \mathrm{~mm}, 0.976562 \mathrm{~mm} ; \mathrm{NEX}=1$. The average total imaging session took approximately $45 \mathrm{~min}$ to complete on each subject. Conscious sedation (Versedt) and anesthesia (Propofol) were administered by a licensed, Board Certified, Anesthesiologist as specified by our IRBapproved Human Subjects protocol. Full written parental consent and child assent (when appropriate) were obtained prior to the MRI procedure.

Utilizing DICOM transfer protocols, the high resolution T1-weighted whole brain image sets from each subject were electronically transferred over a secure network connection from the MRI suite to the DICOM receiver and file server located in the research laboratory. Quantitative comparisons between subjects in computed volumes of whole brain as well as individual substructures were performed. Specifically, a reliable, well-documented, and semi-automated procedure for MR image segmentation (i.e. tissue classification) into gray matter, white matter, and cerebral spinal fluid was implemented according to the protocol detailed elsewhere [31].

Morphometric analysis All scans were analyzed blind to diagnosis, age, and gender. Two technicians served as independent raters prior to a final review by the $2^{\text {nd }}$ author. The inter-rater correlation of sampled MRIs was $>0.85$ for all parcellation data.
Table 1 Sample description for the full sample ( $n=50$, center columns) and for the sample constrained to right-handed males $(n=30$, right sided columns)

\footnotetext{
${ }^{\mathrm{a}}$ standard score
}

\begin{tabular}{|c|c|c|c|c|c|c|}
\hline & \multicolumn{3}{|c|}{ All $(n=50)$} & \multicolumn{3}{|c|}{ Right-handed males $(n=30)$} \\
\hline & $\mathbf{N}$ & Mean & SD & $\mathbf{N}$ & Mean & SD \\
\hline Age at MRI & 50 & 84.4 & 32.4 & 30 & 94.8 & 33.4 \\
\hline FSIQ & 50 & 63.0 & 22.5 & 30 & 62.5 & 21.5 \\
\hline VIQ & 50 & 57.7 & 23.5 & 30 & 56.5 & 22.3 \\
\hline NVIQ & 50 & 68.7 & 22.5 & 30 & 69.4 & 23.3 \\
\hline ADOS social & 50 & 11.2 & 2.0 & 30 & 11.6 & 2.1 \\
\hline ADOS communication & 50 & 6.4 & 1.4 & 30 & 6.6 & 1.2 \\
\hline ADI social & 50 & 24.4 & 3.8 & 30 & 24.9 & 4.2 \\
\hline ADI NV comm & 20 & 12.2 & 2.0 & 10 & 12.6 & 1.1 \\
\hline ADI V comm & 30 & 18.3 & 4.5 & 20 & 18.9 & 4.2 \\
\hline ADI stereotypic behavior & 50 & 6.6 & 2.4 & 30 & 7.3 & 2.4 \\
\hline Vineland adaptive behavior composite $^{a}$ & 50 & 54.4 & 17.3 & 30 & 54.0 & 14.9 \\
\hline Vineland communication $^{\mathrm{a}}$ & 50 & 56.4 & 21.0 & 30 & 55.0 & 19.5 \\
\hline Vineland socialization $^{\mathrm{a}}$ & 50 & 60.4 & 15.4 & 30 & 58.2 & 11.4 \\
\hline Vineland daily living skills ${ }^{\mathrm{a}}$ & 50 & 55.8 & 19.6 & 30 & 57.1 & 17.4 \\
\hline PPVT $^{\mathrm{a}}$ & 32 & 62.9 & 22.1 & 20 & 64.0 & 21.0 \\
\hline EOWPVT $^{\mathrm{a}}$ & 31 & 71.0 & 20.4 & 19 & 68.3 & 18.9 \\
\hline
\end{tabular}


Positional normalization Since validation analyses have documented the significant contribution of positional variability to morphometric analyses, each sagittal $T_{1}$ weighted "native" MRI scan underwent positional normalization prior to anatomic segmentation to reduce these effects without requiring uniformity of head position during the scanning procedure. Specifically, a three-dimensional Cartesian coordinate system was used, referenced to the decussations of the anterior (AC) and posterior (PC) commissures and bisecting the interhemispheric fissure at the level of the PC in the coronal plane [32]. The axes of the coordinate system were: $x$-axis increased laterally from the child's right to left; y-axis increased from posterior to anterior; and z-axis increased from superior to inferior. The reformatted native scan was resliced into normalized $1.20 \mathrm{~mm}$ coronal and $1.0 \mathrm{~mm}$ sagittal scans, which were used for all subsequent morphometric procedures.

Image segmentation and neocortical parcellation

The image analysis method for segmentation has been previously described in detail [32]. Briefly, on each normalized $\mathrm{T}_{1}$-weighted $3 \mathrm{D}$ MRI slice, anatomic segmentation was performed using intensity contour mapping and differential intensity contour algorithms, which identify, classify and create a continuous outline corresponding only to those voxels constituting boundaries between tissue classes, as previously described [31,32]. Such algorithms delineate outline boundaries by recruiting voxels bordering transitional changes in image intensity. The set of global anatomic structures included cortical and subcortical gray matter, white matter, and cerebrospinal fluid (Supplementary Fig. 1). This process results in a segmented neocortical ribbon, which is dissociated from other brain regions and structures.

The neocortical ribbon was parcellated into 48 units in each hemisphere [31, 33, 34]. Boundaries of each parcellation unit are simply defined by referencing a set of standardized landmarks localized on each individual brain. As reported previously, the use of color-coded sulci markers manually traced onto each subject's MRI facilitates the construction of individual neocortical maps (Supplementary Fig. 2). This methodology preserves the inter-individual uniqueness in brain topography (e.g., gyri and fissures) and provides a distinctive basis for evaluating inter-individual variability separate from group variability. Right hemisphere parcellation maps are shown in Supplementary Fig. 3.

The anatomic boundaries for the ROIs (PP, HG, PT, and pSTG) are as previously described [31-33] (Fig. 1): PP: anterior aspect bounded by isthmus of temporal and frontal lobes, posterior aspect bounded by the first transverse fissure of Heschl (posterior limit), medial aspect bounded by circular fissure of insula, lateral aspect bounded by the hemisphere margin of the Sylvian fissure. HG: anterior aspect bounded by anterior limit of first transverse fissure of Heschl, posterior aspect bounded by posterior limit of Heschl's gyrus, medial aspect bounded by circular fissure of the insula, lateral aspect bounded by hemispheric margin of the Sylvian fissure. PT: anterior aspect bounded by Heschl's gyrus (anterior aspect), posterior aspect bounded by posterior limit of the Sylvian fissure, medial aspect bounded by circular fissure of the insula, lateral aspect bounded by hemispheric margin of the Sylvian fissure. pSTG: anterior aspect bounded by anterior limit of first transverse fissure of Heschl's gyrus, posterior aspect bounded by posterior limit of Sylvian fissure, medial aspect bounded by hemispheric margin of the Sylvian fissure, lateral aspect bounded by superior temporal fissure (see Supplementary Figs. 4 and 5 for slice segmentation and parcellation details for one representative subject).

Statistical methods

All analyses were conducted two ways: using the raw volumes and using volumes that were normalized for total brain volume (TBV). Results did not differ when normalized to TBV. We reported raw volumes so that findings are more easily comparable to other studies. Data analyses were primarily descriptive. A Symmetry Index (SI) was calculated as right brain volume minus left brain volume divided by the average of right plus left volume $([2(\mathrm{R}-\mathrm{L}) / \mathrm{R}+\mathrm{L})]$, after Galaburda [35]. Means and 95\% confidence intervals (CIs) were calculated for measures of asymmetry. The influence on SI of gender, handedness, IQ and age at MRI was investigated using analysis of variance and linear regression. Numbers of females and left-handed subjects were small hence there was limited power to detect differences between these groups. Although graphic presentation of the data supports the absence of gender and handedness differences, asymmetry measurements are reported for the restricted group of righthanded males to eliminate any possible confounding.

\section{Results}

Full sample $(n=50)$

The participants studied include 41 males and 9 females (male-female ratio: 4.6). Children averaged 89 months at MRI testing ( $\mathrm{SD}=32.4$, age range $2-14$ years) with mean full-scale, verbal and non-verbal IQ of 63,58 and 69 respectively (Table 1). Children demonstrate moderate impairment in adaptive behavior and language abilities. The Vineland Adaptive Behavior Composite mean score was $54.4(\mathrm{SD}=17.3)$ with domain scores ranging from 54 
Fig. 1 Mean volumes (in units of cubic centimeters (cc) for left and right hemispheres for the unconstrained sample $(n=50)$ : a planum temporale, b planum polare, c Heschl's gyrus, and d posterior superior temporal gyrus.. Error bars depict standard error (SE)

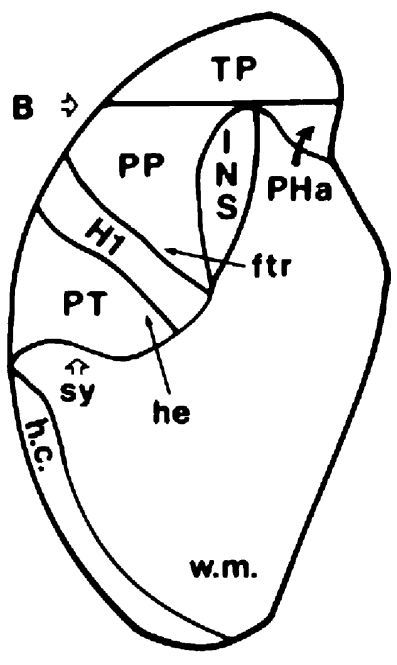

to 60. Standard scores from language tests reflect a similar level of impairment (PPVT mean score $=62.94, \mathrm{SD}=22.1$; EOWPVT mean score=71.0, $\mathrm{SD}=20.4$ ).

Mean volumes in units of cubic centimeters for the four regions of interest in left and right hemispheres are presented in Table 2. SI mean values and 95\% CIs are shown in Table 3. There was an absence of PT asymmetry (Table 2), with mean asymmetry (SI) of 0.06 , with $95 \%$ CI including zero ( -0.01 to 0.13 ); values are distributed above and below zero with the majority of values above zero (Fig. 2). PP results show no significant asymmetry (mean $\mathrm{SI}=-0.02,95 \% \mathrm{CI}:-0.06$ to 0.03$)$. In contrast, HG asymmetry showed notable left-dominance with 95\% CI entirely below zero and the majority of individuals having $\mathrm{LH}>\mathrm{RH}$ (mean $\mathrm{SI}=-0.22,95 \%$ confidence interval -0.30 to $-0.15)$. The majority of children exhibited right-dominance in $\mathrm{pSTG}$, with $\mathrm{RH}>\mathrm{LH}$ (mean $\mathrm{SI}=0.11,95 \% \mathrm{CI}$ : 0.03 to 0.19). Although the numbers of females and left-handed subjects were small, there were no significant differences in asymmetry due to gender or handedness when tested by oneway analysis of variance. Results for MZ twins $(n=6$, all male) show hemisphere asymmetries that differed across the brain ROIs and sets of twins (Supplementary Fig. 6).

Constrained sample (right-handed boys, $n=30$ )

When the sample was restricted to right-handed males to control for gender and handedness, results did not change (Table 3 and Fig. 3). Mean asymmetry measures (and 95\% CIs) for the restricted group show rightdominance for PT and pSTG, left-dominance for $\mathrm{HG}$, and an absence of PP asymmetry. Rightward asymmetry for PT and pSTG were statistically significantly positively correlated $(r=.444, p<.01)$.

Age at scan was significantly associated with PT asymmetry $(r=0.30, p=0.03)$. Right dominance was observed with increasing frequency at older ages compared to younger age at scan (Fig. 4). Age at scan was not significantly associated with the asymmetry index for PP, $\mathrm{HG}$, or pSTG $(r=0.10, r=-0.02$, and $r=0.08$ respectively, $p>$ $0.05)$. No association with IQ was noted for any of the asymmetry measures investigated. No association between neuropsychological language measures and PT or pSTG asymmetry was noted.

\section{Discussion}

We conducted quantitative morphometric analysis of four ROIs to assess hemispheric asymmetries in children with AD. We report several key findings: (i) lack of PT asymmetry in the unconstrained sample, with a trend for a rightward bias, (ii) significantly rightward PT asymmetry when the sample was constrained to right handed boys; (iii) rightward asymmetry of pSTG that was positively correlated with PT; (iv) age dependence of PT asymmetry, with more rightward asymmetry with increasing age; and (v) lack of correspondence between neuropsychological language measures and PT and pSTG asymmetry.

Our PT findings are in general agreement with those of Rojas and colleagues, who reported lack of PT asymmetry in children and adults with $\mathrm{AD}[20,21]$, however our findings differ somewhat from theirs in that we reported significantly rightward PT asymmetry in right-handed boys. Our PT findings differ from those of Herbert and De Fosse and their colleagues [19, 22, 36] who reported leftward PT asymmetries for children with AD. It is important to note that the morphometric analyses in the present investigation were nearly identical to those utilized by De Fosse and Herbert, thus our disparate findings correspond to brain differences across the samples and not methodological differences.
Table 2 Mean and standard error (SE) volumes (in units of cubic centimeters (cc)) for left and right hemispheres for the unconstrained sample $(n=50)$ : a) planum temporale, b) planum polare, c) Heschl's gyrus, and d) posterior superior temporal gyrus

\begin{tabular}{|c|c|c|c|c|}
\hline & \multicolumn{2}{|c|}{ Left hemisphere $(n=50)$} & \multicolumn{2}{|c|}{ Right hemisphere $(n=50)$} \\
\hline & Mean & SE & Mean & SE \\
\hline planum temporale & 1.779 & 0.102 & 1.869 & 0.091 \\
\hline planum polare & 2.581 & 0.076 & 2.521 & 0.067 \\
\hline Heschl's gyrus & 1.666 & 0.078 & 1.327 & 0.060 \\
\hline posterior superior temporal gyrus & 2.859 & 0.162 & 3.174 & 0.149 \\
\hline
\end{tabular}


Table 3 Asymmetry Index for the full sample $(n=50$, center columns) and for the sample constrained to right-handed males $(n=30$, right sided columns)

\begin{tabular}{|c|c|c|c|c|}
\hline & \multicolumn{2}{|c|}{ All $(n=50)$} & \multicolumn{2}{|c|}{ Right-Handed Males $(n=30)$} \\
\hline & Mean & $95 \% \mathrm{CI}$ & Mean & $95 \%$ CI \\
\hline planum temporale & 0.061 & $-0.009,0.131$ & 0.093 & $0.002,0.185$ \\
\hline planum polare & -0.018 & $-0.062,0.025$ & -0.031 & $-0.088,0.025$ \\
\hline Heschl's gyrus & -0.223 & $-0.300,-0.146$ & -0.186 & $-0.286,-0.086$ \\
\hline posterior superior temporal gyrus & 0.114 & $0.0346,0.193$ & 0.145 & $0.048,0.243$ \\
\hline
\end{tabular}

The results of De Fosse and colleagues [22], suggested that degree of PT asymmetry may correspond with level of language impairment, however our findings did not support this hypothesis. The lack of correspondence between PT asymmetry and neuropsychological language measures may reflect the complex relationship between normative measures of cognitive ability and the wide range of behaviors observed in individuals on the autistic spectrum [37, 38]. Further, it has been demonstrated in TD children that IQ may correspond to the developmental trajectory of brain maturation rather than to specific structural measures [39]. If the failure to achieve significant correspondence between neuropsychological tests and brain measures was due to a general lack of sensitivity for neuropsychological tests as predictors of brain structure, one might still expect to find some degree of systematic correspondence between neuro- psychological and brain measures, however this was not the case in our investigation.

We reported significant leftward HG asymmetry in both unconstrained and constrained samples, a finding in good accord with those of Rojas and colleagues [20, 21]. A trend for larger left HG was also reported by De Fosse, Herbert, and their colleagues for children with $\operatorname{AD}[19,22]$. Leftward asymmetry for $\mathrm{HG}$ is a classic finding in the literature $[40,41]$ that has been supported by recent neuroimaging studies $[42,43]$. Thus, our findings combine with those of De Fosse, Herbert and Rojas, to provide evidence for a HG developmental path in $\mathrm{AD}$ that does not diverge from typical [19-22, 36].

We report lack of PP asymmetry in both unconstrained and the constrained samples. Herbert and colleagues [19] reported no group effect (AD vs. TD) for PP, however did

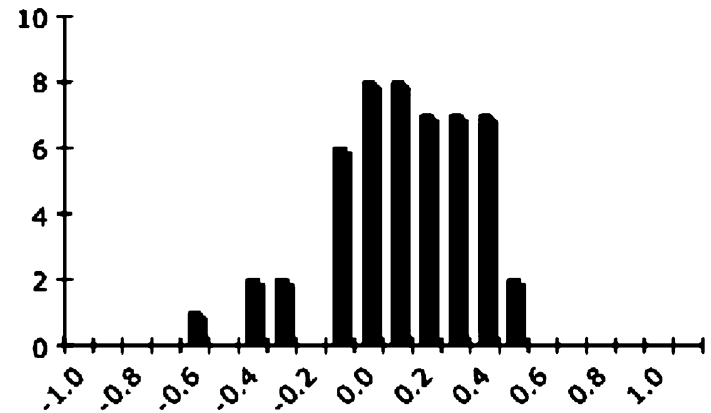

\section{a. planum temporale}

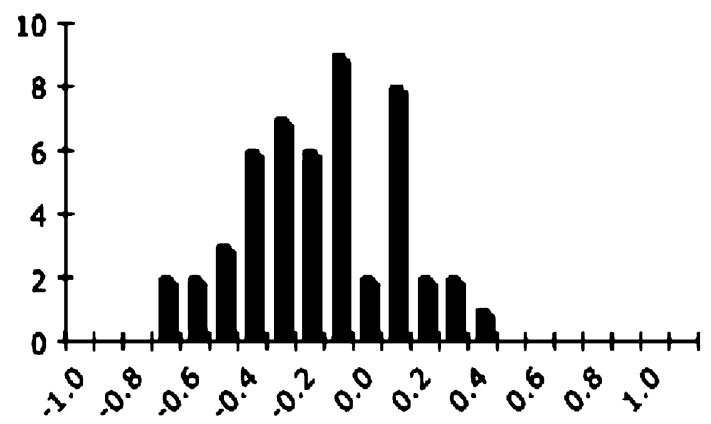

\section{c. Heschl's gyrus}

Fig. 2 Coefficent of asymmetry histogram for the unconstrained sample $(n=50)$ : a planum temporale, b planum polare, c Heschl's gyrus, and $\mathbf{d}$ posterior superior temporal gyrus. The coefficient was

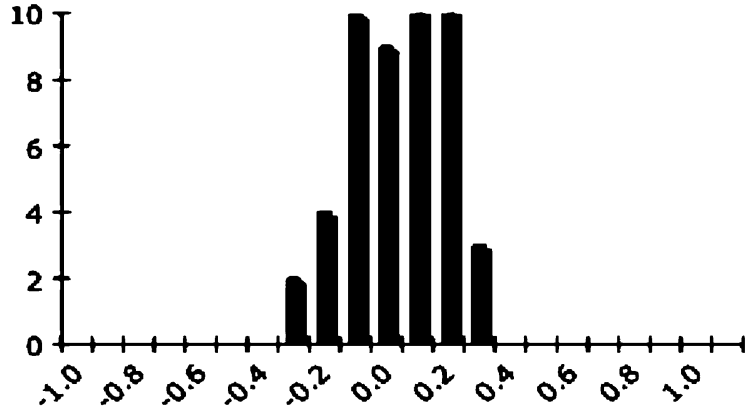

b. planum polare

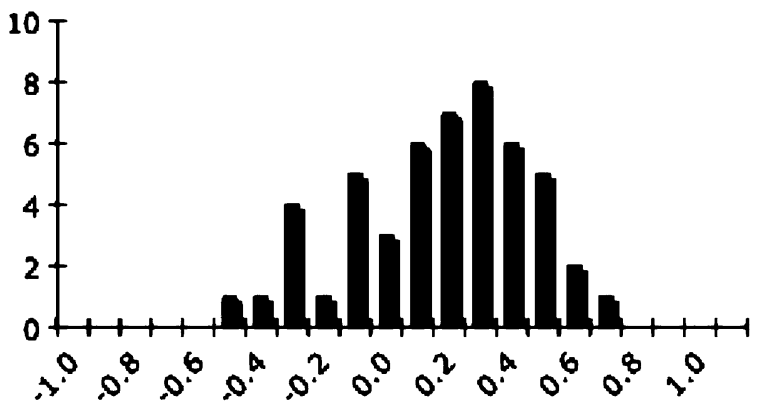

d. PSTG

calculated as $([2(\mathrm{R}-\mathrm{L}) / \mathrm{R}+\mathrm{L})]$, after [35]), and values range from -1.0 to 1.0 , with negative values reflecting leftward asymmetry 


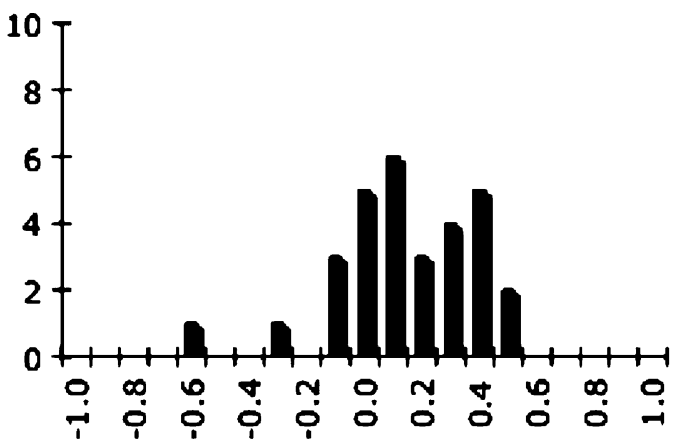

\section{a. planum temporale}

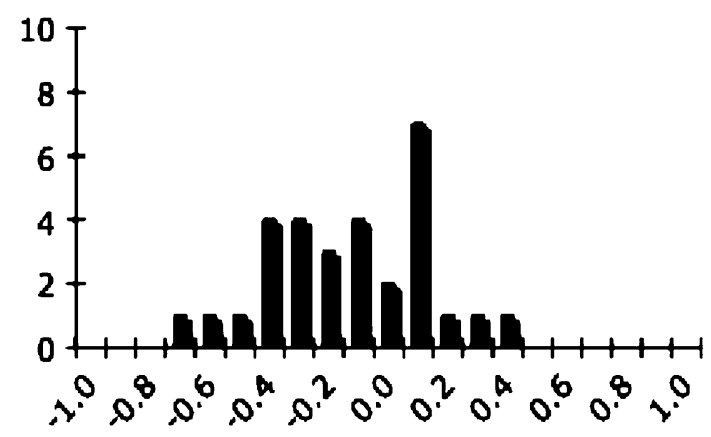

\section{c. Heschl's gyrus}

Fig. 3 Coefficent of asymmetry histogram for the constrained sample of right-handed males $(n=30)$ : a planum temporale, $\mathbf{b}$ planum polare, c Heschl's gyrus, and d posterior superior temporal gyrus. Coefficient

not report whether the PP was symmetric or not; and it was not assessed in the De Fosse and Rojas studies. Little is known about PP asymmetries in neurotypicals, however an aMRI parcellation study by Kennedy and colleagues reported no PP asymmetries in a sample of young adults [33], corresponding to our results.

We report a rightward pSTG asymmetry for both the unconstrained and constrained samples. These results differ from those of Herbert and De Fosse and colleagues [19, 22] who reported no reliable group differences in pSTG: there was a non-statistically significant trend for leftward asymmetry for children with $\mathrm{AD}$ and rightward asymmetry for controls in the Herbert et al. study, however the distributions were largely overlapping. A similar effect was seen in the de Fosse et al. study, however in this case there was a trend for rightward asymmetry for children with $\mathrm{AD}$ and leftward asymmetry for controls, and again the distributions were overlapping. Difference across studies may reflect the much lower cognitive function of our larger sample. Our sample, at 50 children, is quite large for a neuroimaging study and fairly homogenous: all children met research criteria for idiopathic autistic disorder and had no chromosomal abnormalities (based upon previous genetic studies). It may be the case that the relatively low

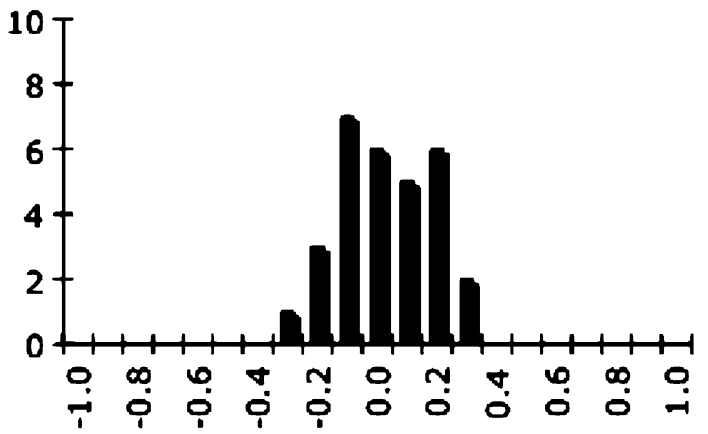

b. planum polare

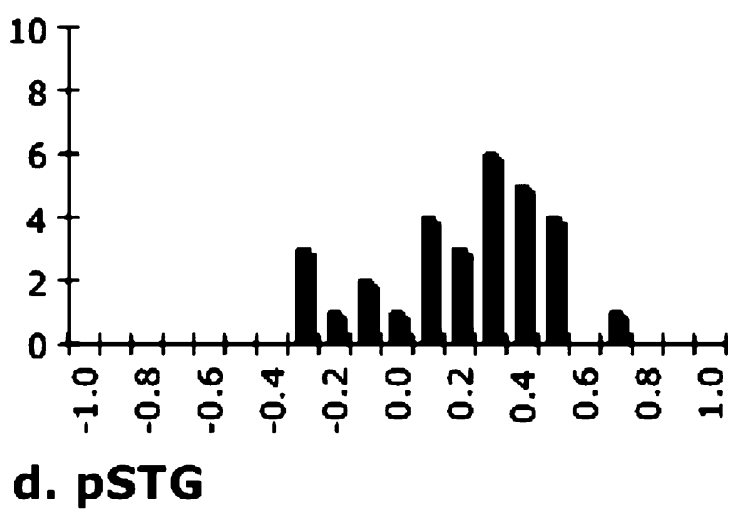

values range from -1.0 to 1.0 , with negative values reflecting leftward asymmetry

cognitive and language functioning of the group had an impact on our results. The fact that we found a significant rightward pSTG asymmetry in our sample whose Verbal IQ averaged below 60 (M 57.7 SD 23.5) - an opposite effect to those found by De Fosse and Herbert and their colleagues with a higher functioning sample - may reflect differing neuroanatomical substrates for lower- vs. higherfunctioning individuals with $\mathrm{AD}$.

An open question is whether the rightward asymmetries we found in both pSTG and PT correspond to rightward frontal lobe asymmetries found for the higher functioning children in the Herbert and de Fosse studies [19, 22]. The rightward asymmetries in pSTG and PT found in our sample and the rightward frontal lobe asymmetries reported in the studies of Herbert and de Fosse may correspond to reports of rightward functional asymmetry in $\mathrm{AD}[4,10-12$, 14-17, 44-47]. The functional region known as Wernicke's area lies within the boundaries of PT and pSTG and both regions show activation for language stimuli, although it is becoming increasingly clear that PT is not a languagespecific region [48]. Our findings of a significant positive correlation between the symmetry indices for PT and pSTG is not surprising given that together they form the anatomical substrate of the functional Wernicke's area, 
Fig. 4 Relationship between age and asymmetry for the unconstrained sample $(n=50)$ : a planum temporale, b planum polare, c Heschl's gyrus, and d) posterior superior temporal gyrus. Y-axis depicts brain volume (cc) and $\mathrm{x}$-axis depicts age at MRI scan in months
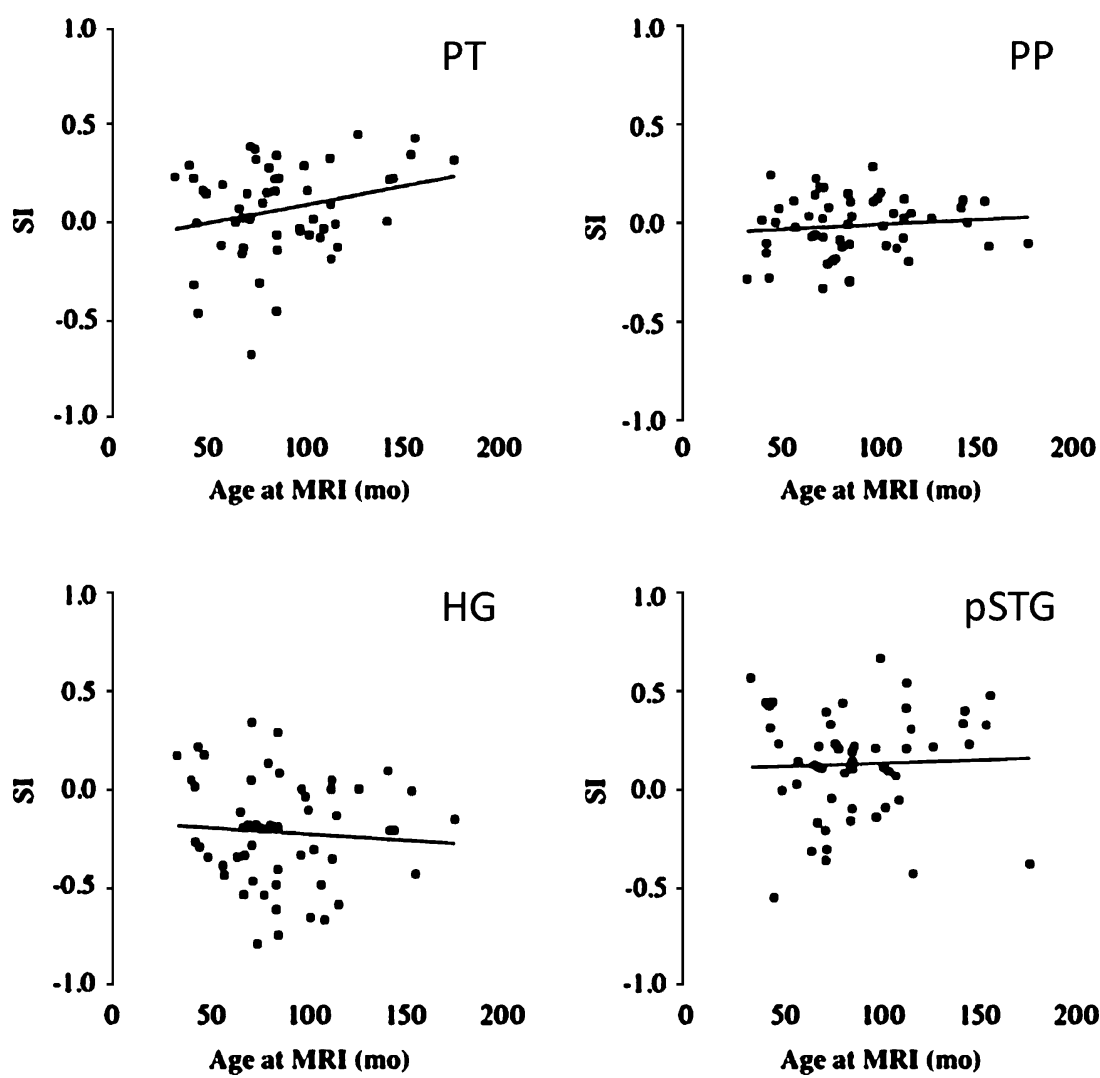

and suggests a relationship in the maturational paths of these two auditory language association regions.

We report PT age dependence, with increased rightward asymmetry with age. Asymmetries for $\mathrm{HG}, \mathrm{PP}$, and pSTG did not correspond with age, indicating that there are no gross structural maturational changes occurring in these regions for children in the age range (2-14 years) studied here. Age-related anatomical changes have been reported for TD children in the perisylvian region, with increased hemispheric asymmetries with age [49]. The authors suggested that these results might be due to cellular-based maturational processes such as synaptic pruning and myelination. However, our findings of age-dependence are restricted to the $\mathrm{PT}$, with asymmetries for $\mathrm{HG}$, $\mathrm{PP}$, and pSTG showing no significant age correspondence, therefore it is unlikely that they reflect general cortical maturational processes. Clearly more studies are needed, both in typical and atypical development, in order to shed light on maturational changes in the perisylvian cortex.

In a small $(n=6)$ sample of MZ twins, we report lack of concordance in asymmetries in each of the ROIs, leaving open the question of epigenetic factors affecting hemispheric asymmetries (Supplementary Fig. 6). This notion is supported by reports of lack of concordance for PT asymmetries in typically developing $\mathrm{MZ}$ twins, where lack of concordance across sets of twins has been suggested to relate to differential action of nongenetic factors in twin pairs [50, 51].
In summary, we provide evidence that $\mathrm{HG}, \mathrm{PP}$, and pSTG show asymmetry patterns similar to previous findings for $\mathrm{AD}$ and $\mathrm{TD}$ children. PT asymmetry showed a different pattern, with rightward asymmetry for righthanded boys that differs from some previous findings for children with $\mathrm{AD}$ and for classical and current findings in neurotypicals [18, 50, 52-55]. PT asymmetry did not correspond to neuropsychological measures of language ability but did correspond with age, indicating that experience may influence PT asymmetries in children with AD. This notion is further supported by lack of concordance of PT asymmetry in sets of MZ twins. Our PT results for relatively low functioning children with $\mathrm{AD}$ represent the largest study of its kind to date. The general agreement between our findings and those of Rojas and colleagues [20] provides clear evidence that some children with AD have a lack of leftward bias in this strongly left lateralized brain region. This sample of children is lower functioning and represents a larger proportion of children with $\mathrm{AD}$ then the previously published smaller studies looking specifically at high functioning individuals with AD. Our findings of age dependence of PT asymmetry suggest that there may be interactions between PT structural development and auditory language experience. In conclusion, our finding of rightward PT asymmetry provides evidence for a different maturational path in $\mathrm{AD}$ for this highly lateralized brain structure. 
Acknowledgements We are grateful to the children and their families for volunteering to participate in this research. This research was funded by the National Institutes of Health (PO1HD 35458 to MAS); The National Alliance for Autism Research (177 to NMG).

Open Access This article is distributed under the terms of the Creative Commons Attribution Noncommercial License which permits any noncommercial use, distribution, and reproduction in any medium, provided the original author(s) and source are credited.

\section{References}

1. Kanner L. Autistic disturbances of affective contact. Nerv Child. 1943;2:217-50.

2. Branch C, Milner B, Rasmussen T. Intracarotid sodium amytal for the lateralization of cerebral speech dominance; observations in 123 patients. J Neurosurg. 1964;21:399-405.

3. Dehaene-Lambertz G, Hertz-Pannier L, Dubois J. Nature and nurture in language acquisition: anatomical and functional brainimaging studies in infants. Trends Neurosci. 2006;29:367-73.

4. Flagg EJ, Cardy JE, Roberts W, Roberts TP. Language lateralization development in children with autism: insights from the late field magnetoencephalogram. Neurosci Lett. 2005;386:82-7.

5. Holland SK, Plante E, Weber Byars AM, Strawsburg RH, Schmithorst VJ, et al. Normal brain activation patterns in children performing a verb generation task. Neuroimage. 2001;14:837-43.

6. Balsamo LM, Xu B, Grandin CB, Petrella JR, Braniecki SH, Elliott TK, et al. A functional magnetic resonance imaging study of left hemisphere language dominance in children. Arch Neurol. 2002;59:1168-74.

7. Gaillard WD, Balsamo LM, Ibrahim Z, Sachs BC, Xu B. fMRI identifies regional specialization of neural networks for reading in young children. Neurology. 2003;60:94-100.

8. Holland SK, Vannest J, Mecoli M, Jacola LM, Tillema J-M, Kakunanayaka PR, et al. Functional MRI of language lateralization during developing in children. Intl J of Aud. 2007;46:533-51.

9. Prior MR, Bradshaw JL. Hemisphere functioning in autistic children. Cortex. 1979;15:73-81.

10. Chiron C, Leboyer M, Leon F, Jambaque I, Nuttin C, Syrota A. SPECT of the brain in childhood autism: evidence for a lack of normal hemispheric asymmetry. Dev Med Child Neurol. 1995;37:849-60.

11. Muller RA, Behen ME, Rothermel RD, Chugani DC, Muzik O, Mangner TJ, et al. Brain mapping of language and auditory perception in high-functioning autistic adults: a PET study. J Autism Dev Disord. 1999;29:19-31.

12. Ohnishi T, Matsuda H, Hashimoto T, Kunihiro T, Nishikawa M, Uema $\mathrm{T}$, et al. Abnormal regional cerebral blood flow in childhood autism. Brain. 2000;123:1838-44.

13. Escalante-Mead PR, Minshew NJ, Sweeney JA. Abnormal brain lateralization in high-functioning autism. J Autism Dev Disord. 2003;33:539-43.

14. Boddaert N, Chabane N, Belin P, Bourgeois M, Royer V, Barthelemy $\mathrm{C}$, et al. Perception of complex sounds in autism: abnormal auditory cortical processing in children. Am J Psychiatry. 2004;161:2117-20.

15. Just MA, Cherkassky VL, Keller TA, Minshew NJ. Cortical activation and synchronization during sentence comprehension in high-functioning autism: evidence of underconnectivity. Brain. 2004; $127: 1811-21$.

16. Takeuchi M, Harada M, Matsuzaki K, Nishitani H, Mori K. Difference of signal change by a language task on autistic patients using functional MRI. J Med Invest. 2004;51:59-62.
17. Harris GJ, Chabris CF, Clark J, Urban T, Aharon I, Steele S, et al. Brain activation during semantic processing in autism spectrum disorders via functional magnetic resonance imaging. Brain Cogn. 2006;61:54-68.

18. Shapleske J, Rossell SL, Woodruff PW, David AS. The planum temporale: a systematic quantitative review of its structural functional and clinical significance. Brain Res Brain Res Rev. 1999;29:26-49.

19. Herbert MR, Harris GJ, Adrien KT, Ziegler DA, Makris N, Kennedy DN, et al. Abnormal asymmetry in language association cortex in autism. Ann Neurol. 2002;52:588-96.

20. Rojas DC, Camou SL, Reite ML, Rogers SJ. Planum temporale volume in children and adolescents with autism. J Autism Dev Disord. 2005;35:479-86.

21. Rojas DC, Bawn SD, Benkers TL, Reite ML, Rogers SJ. Smaller left hemisphere planum temporale in adults with autistic disorder. Neurosci Lett. 2002;328:237-40.

22. De Fosse L, Hodge SM, Makris N, Kennedy DN, Caviness CV Jr, McGrath L, et al. Language-association cortex asymmetry in autism and specific language impairment. Ann Neurol. 2004;56: 757-66.

23. Lord C, Risi S, Lambrecht L, Cook EH Jr, Leventhal BL, DiLavore PC, et al. The autism diagnostic observation schedulegeneric: a standard measure of social and communication deficits associated with the spectrum of autism. J Autism Dev Disord. 2000;30:205-23.

24. Lord C, Pickles A, McLennan J, Rutter M, Bregman J, Folstein S, et al. Diagnosing autism: analyses of data from the autism diagnostic interview. J Autism Dev Disord. 1997; 27:501-17.

25. Oldfield RC. The assessment and analysis of handedness: the Edinburgh inventory. Neuropsychologia 1971;97-113.

26. Thorndike RL, Hagen ER, Sattler JM. The stanford-binet intelligence scale (4th ed). Chicago: Riverside; 1986.

27. Mullen EM. Mullen scales of early learning (AGS ed). Circle Pines: American Guidance Services Inc.; 1995.

28. Sparrow SS, Balla DA, Cicchetti DV. Vineland adaptive behavior scales: interview edition survey form manual. Circle Pines: American Guidance Service; 1984.

29. Gardner M. Expressive one-word picture vocabulary test-revised. Novato: Academic Therapy; 1983.

30. Dunn LM, Dunn LM. Peabody picture vocabulary test (PPVT-III). Circle Pines: American Guidance Service; 1997.

31. Caviness CV Jr, Kennedy DN, Richelme C, Rademacher J, Filipek PA. The human brain age 7-11 years: a volumetric analysis based on magnetic resonance images. Cereb Cortex. 1996;6:726-36.

32. Filipek PA, Richelme C, Kennedy DN, Caviness VC Jr. The young adult human brain: an MRI-based morphometric analysis. Cereb Cortex. 1994;4:344-60.

33. Kennedy DN, Lange N, Makris N, Bates J, Meyer J, Caviness VS Jr. Gyri of the human neocortex: an MRI-based analysis of volume and variance. Cereb Cortex. 1998;8:372-84.

34. Rademacher J, Galaburda AM, Kennedy DN, Filipek PA, Caviness VS Jr. Human cerebral cortex: localization parcellation and morphometry with magnetic resonance imaging. J Cogn Neurosci. 1992;4(4):352-84.

35. Galaburda AM, Sanides F, Geschwind N. Human brain. cytoarchitectonic left-right asymmetries in the temporal speech region. Arch Neurol. 1978;35:812-7.

36. Herbert MR, Ziegler DA, Deutsch CK, O’Brien LM, Kennedy $\mathrm{DN}$, Filipek PA, et al. Brain asymmetries in autism and developmental language disorder: a nested whole-brain analysis. Brain. 2005;128:213-26.

37. Happe F. Autism: cognitive deficit or cognitive style? Trends Cogn Sci. 1999;3:216-22. 
38. Joseph RM, Tager-Flusberg H, Lord C. Cognitive profiles and social-communicative functioning in children with autism spectrum disorder. J Child Psychol Psychiatry. 2002;43:807-21.

39. Shaw P, Lerch J, Greenstein D, Sharp W, Clasen L, Evans A, et al. Longitudinal mapping of cortical thickness and clinical outcome in children and adolescents with attention-deficit/hyperactivity disorder. Arch Gen Psychiatry. 2006;63:540-9.

40. Heschl R. Uber die vordere quere schlafendwindung des menschlichen grosshirns. Vienna: Braumuller; 1878.

41. von Economo C, Horn L. Uber windungsrelief mabe und rindenarchitektonic der supratemparalflache ihre individuellen und seitenunterschiede. Neuropsychiatre. 1930;30:678-757.

42. Penhune VB, Zatorre RJ, MacDonald JD, Evans AC. Interhemispheric anatomical differences in human primary auditory cortex: Probabilistic mapping and volume measurement from magnetic resonance scans. Cereb Cortex. 1996;6:661-72.

43. Dorsaint-Pierre R, Penhune VB, Watkins KE, Neelin P, Lerch JP, Bouffard $\mathrm{M}$, et al. Asymmetries of the planum temporale and heschl's gyrus: relationship to language lateralization. Brain. 2006;129:1164-76.

44. Dawson G, Finley C, Phillips S, Galpert L. Hemispheric specialization and the language abilities of autistic children. Child Dev. 1986;57:1440-53.

45. Dawson G, Finley C, Phillips S, Lewy A. A comparison of hemispheric asymmetries in speech-related brain potentials of autistic and dysphasic children. Brain Lang. 1989;37:26-41.

46. Kemner C, Verbaten MN, Cuperus JM, Camfferman G, van Engeland H. Auditory event-related brain potentials in autistic children and three different control groups. Biol Psychiatry. 1995;383:150-65.
47. Bruneau N, Roux S, Adrien JL, Barthelemy C. Auditory associative cortex dysfunction in children with autism: evidence from late auditory evoked potentials (N1 wave-T complex). Clin Neurophysiol. 1999;110:1927-34.

48. Eckert MA, Leonard CM, Possing ET, Binder JR. Uncoupled leftward asymmetries for planum morphology and functional language processing. Brain Lang. 2006;98:102-11.

49. Sowell ER, Thompson PM, Rex D, Komsand D, Tessner KD, Jernigan TL, et al. Mapping sulcal pattern asymmetry and local cortical surface gray matter distribution in vivo: Maturation in perisylvian cortices. Cereb Cortex. 2002;12:17-26.

50. Steinmetz H. Structure functional and cerebral asymmetry: in vivo morphometry of the planum temporale. Neurosci Biobehav Rev. 1996;20:587-91.

51. Eckert MA, Leonard CM, Molloy EA, Blumenthal J, Zijdenbos A, Giedd JN. The epigeneiss of planum temporale asymmetry in twins. Cereb Cortex. 2002;12:749-55.

52. Chi JG, Dooling EC, Gilles F. Left-right asymmetries of the temporal speech areas of the human foetus. Arch Neurol. 1977;34:340-8.

53. Preis S, Jancke L, Schmitz-Hillebrecht J, Steinmetz H. Child age and planum temporale asymmetry. Brain Cogn. 1999;40:441-52.

54. Wada J, Clarke R, Hamm A. Cerebral hemispheric asymmetry in humans. cortical speech zones in 100 adult and 100 infant brains. Arch Neurol. 1975;32:239-46.

55. Watkins KE, Paus T, Lerch JP, Zijdenbos A, Collins DL, Neelin P, et al. Structural asymmetries in the human brain: a voxel-based statistical analysis of 142 MRI scans. Cereb Cortex. 2001;11:86877. 